\title{
RIGIDEZ ARTERIAL - UM NOVO FATOR DE RISCO CARDIOVASCULAR
}

\author{
ARTERIAL STIFFNESS - A NOVEL CARDIOVASCULAR RISK FACTOR
}

Adriana Camargo Oliveira, ${ }^{1,2}$, Weimar Kunz Sebba Barroso,

\section{RESUMO}

A avaliação precisa do risco cardiovascular é essencial para a tomada de decisão clínica. O objetivo do tratamento da hipertensão arterial é reduzir a morbimortalidade, prevenindo danos aos órgãos-alvo. As lesões subclínicas consistem em uma apresentação precoce da doença cardiovascular sem manifestações clínicas aparentes. Os eventos cardiovasculares futuros têm sido tradicionalmente previstos utilizando escores que combinam fatores de risco para aterosclerose convencionais. O aumento da velocidade da onda de pulso (VOP) é um biomarcador padrão-ouro do enrijecimento arterial, e pode avaliar o risco cardiovascular prospectivo e de mortalidade, além de identificar danos aos órgãos e orientar início de tratamento precoce. Efetivamente, os valores da VOP podem reclassificar o risco cardiovascular para um valor mais alto, especialmente em indivíduos mais jovens com risco intermediário. A idade vascular pode ser maior que a idade cronológica, devido aos fatores de risco. O envelhecimento arterial pode resultar em três padrões atualmente estudados: EVA (early vascular aging) - indivíduos com envelhecimento arterial precoce; HVA (healthy vascular aging) - indivíduos com envelhecimento arterial saudável e SUPERNOVA - indivíduos com envelhecimento arterial extremamente baixo para a sua idade. Assim, a avaliação do enveIhecimento vascular fundamentado somente nos fatores de risco cardiovasculares pode falhar, enquanto a VOP representa o dano cumulativo de todos esses fatores na parede arterial. No entanto, existe uma lacuna entre o potencial benefício clínico da avaliação da rigidez arterial e a prática de subutilização no mundo real. Ferramentas clínicas vem sendo desenvolvidas para prever níveis elevados de VOP e identificar pacientes que devem ter a rigidez arterial avaliada.

Descritores: Rigidez Vascular; Análise de Onda de Pulso; Hipertensão; Fatores de Risco; Remodelação Vascular.

\section{ABSTRACT}

Accurate cardiovascular risk assessment is essential for clinical decision-making. The purpose of treating hypertension is to reduce morbidity and mortality, preventing damage to target organs. Subclinical lesions consist of an early presentation of cardiovascular disease with no apparent clinical manifestations. Future cardiovascular events have traditionally been predicted using scores that combine conventional risk factors for atherosclerosis. Increased pulse wave velocity (PWV) is a gold standard biomarker of arterial stiffness, and can assess prospective cardiovascular risk and mortality, identify organ damage and guide early treatment. In fact, PWV can reclassify cardiovascular risk to a higher value, especially in younger individuals with intermediate risk. Vascular age may be greater than chronological age due to risk factors. Arterial aging can result in three patterns currently under study: EVA (early vascular aging) — individuals with early arterial aging; HVA (healthy vascular aging) — individuals with healthy arterial aging and SUPERNOVA — individuals with extremely low arterial aging for their age. Thus, the evaluation of vascular aging solely based on cardiovascular risk factors may fail, while PWV represents the cumulative damage of all these factors on the arterial wall. However, there is a gap between the potential clinical benefit of assessing arterial stiffness and underutilization in the real world. Clinical tools have been developed to predict high levels of PWV and to identify patients who should have their arterial stiffness assessed.

Keywords: Vascular Stiffness; Pulse Wave Analysis; Hypertension; Risk Factors; Vascular Remodeling.

A avaliação precisa do risco cardiovascular (CV) é essencial para a tomada de decisão clínica. O objetivo principal do tratamento da hipertensão arterial (HAS) é prevenir danos em órgãos alvo e reduzir a morbimortalidade cardiovascular. As lesões subclínicas consistem em uma apresentação precoce da doença cardiovascular (DCV) sem manifestações clínicas aparentes. Muitas pontuações / escores foram desenvolvidos ao longo de anos para classificar os pacientes em grupos de risco CV baixo, moderado ou alto. ${ }^{1}$

1. Programa de Pós-Graduação em Ciências da Saúde - Universidade Federal de Goiás.

2. Liga de Hipertensão Arterial - Universidade Federal de Goiás.

Correspondência: Universidade Federal de Goiás, Câmpus Colemar Natal e Silva (Câmpus I) - R. 235, s/n - Setor Leste Universitário, Goiânia - GO, 746O5-O5O sebbarroso@gmail.com

http://dx.doi.org/10.47870/1519-7522/2O2O270113-7 
Na Europa, o HeartScore e sua versão online, Systemic Coronary Risk Evaluation (SCORE), foram desenvolvidos para a previsão do risco cardiovascular total fatal em 10 anos. ${ }^{1,2}$ Nos EUA, foram validados vários escores de risco diferentes. O mais utilizado é originado do grupo Framingham Heart Study. ${ }^{1,3}$ No entanto, o escore recentemente proposto pela American College of Cardiology / American Heart Association (ACC / AHA) tem sido alvo de controvérsias. ${ }^{1,4}$ No Brasil, o escore de risco mais aplicado é o Escore de Risco Global (ERG), que estima o risco de infarto do miocárdio, acidente vascular encefálico (AVE), ou insuficiência cardíaca, fatais ou não fatais, ou insuficiência vascular periférica em 10 anos. $^{5}$

Ferramentas adicionais para estratificar com maior acurácia e de forma precoce o risco dos pacientes são biomarcadores. De acordo com a definição do National Institutes of Health, um biomarcador é "uma característica que é objetivamente medida e avaliada como um indicador da normalidade do processo biológico, patogênico ou resposta farmacológica a uma intervenção terapêutica" ${ }^{1,6}$ Em essência, e no cenário de prevenção, os biomarcadores CV refletem precocemente funções morfológicas ou mudanças, muito antes da manifestação da doença. Podem ser particularmente úteis nos pacientes classificados como risco CV intermediário, em quem há um dilema terapêutico, ou seja, reclassificando-os em um valor de risco mais alto. ${ }^{1}$

O aumento da velocidade da onda de pulso carotídeo-femoral (VOPcf) é a marca registrada do enrijecimento arterial e sugere-se que seja um dos melhores biomarcadores disponíveis para calcular o risco cardiovascular prospectivo e o risco de mortalidade de um indivíduo. ${ }^{7-12}$ Essa identificação da doença subclínica pode abrir uma janela de oportunidade para prevenir a ocorrência de doença CV clínica com a adoção de tratamento precoce e oportuno. ${ }^{1}$

\section{A RIGIDEZ ARTERIAL NA ESTRATIFICAÇÃO DE RISCO CARDIOVASCULAR}

Nas duas últimas décadas, evidências demonstraram que a rigidez arterial é um forte preditor independente de doenças cardiovasculares (DCV) e de mortalidade cardiovascular total, e que é representativa do envelhecimento vascular. ${ }^{7-13}$

A rigidez arterial é um fator preditor da incidência de hipertensão arterial, que por sua vez aumenta a rigidez arterial, em um ciclo vicioso. Isso foi demonstrado pela primeira vez em adultos de meia idade (60 anos) que participaram do Framingham Heart Study ${ }^{7,14}$ e, mais recentemente, em adultos finlandeses normotensos mais jovens (30 a 45 anos). ${ }^{7,15} \mathrm{Em}$ ambos os casos, a VOPcf melhorou a previsão da incidência de hipertensão arterial, muito além fatores de riscos cardiovasculares tradicionais. ${ }^{7,14,15} \mathrm{O}$ estudo Baltimore também demonstrou uma associação entre o aumento da VOPcf e uma maior incidência de hipertensão arterial. ${ }^{16,17}$

Além disso, em revisão sistemática que incluiu 16 estudos com 17.635 participantes, a utilização da VOPcf reclassificou aproximadamente $13 \%$ dos indivíduos para risco intermediário e foi capaz de melhorar a avaliação do prognóstico de risco cardiovascular em 10 anos. ${ }^{12,16}$

Em outro estudo, OPTIMO que incluiu 1416 participantes de 14 países da América Latina, a incidência de envelhecimento arterial precoce foi de $6 \%$, com taxas mais altas em adultos jovens (18,7\% naqueles com 30 anos ou menos). Esses resultados apoiariam a busca de fatores de risco cardiovasculares e envelhecimento arterial precoce no início da fase adulta. ${ }^{18}$

Estudos têm demonstrado que a adição de VOPcf aos fatores de risco tradicionais envolvidos na pontuação de escores, como Framingham e SCORE, aumenta significativamente o valor preditivo para eventos cardiovasculares. $.16,19,20$

Um aumento na VOP aórtica em $1 \mathrm{~m} / \mathrm{s}$ correspondeu a um aumento de 14\%, 15\% e 15\% no total de eventos $\mathrm{CV}$, mortalidade $\mathrm{CV}$ e mortalidade por todas as causas, respectivamente, segundo revisão sistemática que incluiu 15.877 participantes. ${ }^{11}$

\section{A RIGIDEZ ARTERIAL DAS GRANDES ARTÉRIAS E AS LESÕES DE ÓRGÃOS-ALVO}

As alterações vasculares estruturais observadas em pacientes com hipertensão arterial essencial estão comumente relacionadas ao remodelamento eutrófico, em que ocorre um aumento da relação da média/lúmen, sem variação significativa na área transversal da camada média.,21-26

De fato, a remodelação eutrófica pode representar um mecanismo de proteção contra um aumento da pressão arterial no nível da microcirculação. Por outro lado, após vários anos de doença hipertensiva, a estimulação crônica da angiotensina II e endotelina-1 (ET-1) podem resultar no crescimento de células musculares lisas vasculares, levando a um remodelamento hipertrófico. ${ }^{21-26}$

O fenômeno de envelhecimento arterial compreende as alterações relacionadas com uma diminuição da elasticidade arterial e consequente aumento dos níveis de rigidez arterial e a pressão sanguínea sistólica (PAS). Do ponto de vista fisiopatológico, antes do dano endotelial, ocorre a diminuição da quantidade de elastina e aumento da quantidade de colágeno na parede médio-intimal das artérias. ${ }^{21-26}$

O remodelamento das artérias leva ao aumento da resistência periférica, perda de elasticidade, enrijecimento vascular e alterações estruturais nas grandes artérias. ${ }^{21-26}$

As artérias sistêmicas normalmente exercem uma poderosa função de amortecimento, e fornecem o fluxo constante para a microvasculatura apesar da ejeção intermitente do ventrículo esquerdo (VE). Essa função de amortecimento fica prejudicada com o enrijecimento das grandes artérias, levando a múltiplas consequências que têm um grande impacto na saúde cardiovascular. ${ }^{25}$

Primeiro, o enrijecimento das grandes artérias (EGA) causa hipertensão sistólica isolada caracterizada por pressão arterial sistólica aumentada com pressão arterial diastólica normal ou baixa (isto é, pressão de pulso aumentada). ${ }^{25}$

Segundo, o EGA reduz a pressão de perfusão coronariana e aumenta a pós-carga do VE, promovendo remodelamento, disfunção e falha do VE, mesmo na ausência de doença arterial coronariana. ${ }^{25}$

Terceiro, devido ao seu impacto na pulsatilidade da pressão e do fluxo, o EGA promove maior penetração da energia pulsátil na microvasculatura dos órgãos-alvo, particularmente naqueles que requerem alto fluxo sanguíneo e, portanto, devem operar com baixa resistência arteriolar. ${ }^{25}$ 
Embora o enrijecimento da parede arterial anteceda a hipertensão sistólica isolada e contribua causalmente para o dano ao órgão alvo, a própria parede arterial é um órgão alvo, profundamente afetado pelo envelhecimento e por vários estados patológicos, incluindo diabetes, obesidade, tabagismo, hipercolesterolemia e doença renal crônica (DRC). Portanto, o enrijecimento das grandes artérias desempenha um papel central em um ciclo vicioso de disfunção hemodinâmica, caracterizado por excesso de pulsatilidade, o que acaba contribuindo para insuficiência cardíaca (IC), perfusão coronariana prejudicada, DRC, doença cerebrovascular e outras condições crônicas. ${ }^{21-26}$

\section{CONCEITOS DE EXTREMOS NO ENVELHECIMENTO ARTERIAL}

O envelhecimento arterial pode resultar em três padrões atualmente estudados: EVA (early vascular aging) - indivíduos com envelhecimento arterial precoce; HVA (healthy vascular aging) - indivíduos com envelhecimento arterial saudável e SUPERNOVA - indivíduos com envelhecimento arterial extremamente baixo para a sua idade. ${ }^{7}$

O envelhecimento resulta no enrijecimento da parede arterial, que ocorre em paralelo à incidência de hipertensão arterial, seguido de danos subclínicos nos órgãos-alvos e de complicações cardiovasculares de maneira mais acentuada para alguns indivíduos (EVA) do que em outros (SUPERNOVA). ${ }^{7}$

Os indivíduos EVA alcançam cada uma dessas etapas mais cedo que a população média. Por exemplo, a obesidade é um fator que contribui para a mudança ascendente de um padrão de envelhecimento vascular para outro, ou seja, são fatores de risco que favorecem o EVA.7,27

Por outro lado, os indivíduos SUPERNOVA permanecem protegidos por um longo período. O EVA pode ser diagnosticado em pacientes que apresentam rigidez arterial anormalmente alta para idade e sexo. Assim, o EVA representa uma capacidade alterada para reparar danos arteriais em resposta a agressores como estresse mecânico e estresse metabólico / químico / oxidativo. Em outras palavras, o enrijecimento arterial detectado através do aumento da VOPcf é um integrador de todos os danos causados à parede arterial. Além disso, a rigidez aórtica, como marcador de dano da parede arterial ou arteriosclerose, integra tanto o efeito quanto a suscetibilidade a esses fatores de risco. ${ }^{7,27}$

A VOPcf não mede apenas o dano arterial atual (um produto da idade, fatores de risco e suscetibilidade intrínseca a eles), mas também sua regressão (quando uma ação terapêutica é iniciada) ou progressão (quando a exposição continua ou as ações terapêuticas falham). ${ }^{7,23,27}$

Estudos transversais e longitudinais recentes ampliaram a lista dos determinantes epidemiológicos da rigidez arterial. A maioria desses determinantes pertence aos fatores de risco clássicos, não modificáveis, como: etnia, sexo, idade cronológica, histórico familiar e histórico pessoal ou modificáveis, como pressão arterial, diabetes mellitus, dislipidemia e tabagismo. ${ }^{7,28,29}$ Como a rigidez arterial e pressão arterial elevada estão interconectadas, é difícil separar essas entidades como causa ou consequência do EVA. ${ }^{7,26}$

O estabelecimento de um fenótipo vascular extremo
(EVA ou SUPERNOVA) é, portanto, o produto da interação entre (1) as mudanças estruturais na parede arterial que geralmente estão associadas à idade e (2) os mecanismos que aceleram ou desaceleram esse processo, respectivamente. Apesar desses mecanismos, a PA elevada não é uma condição exclusiva para o EVA. Por exemplo, o EVA pode ser causado por inflamação crônica de baixo grau devido à doença inflamatória intestinal, em pacientes que apresentam alta VOP apesar da pressão arterial normal. ${ }^{7}$

Estudos adicionais revelaram o papel da hiperglicemia, síndrome metabólica, resistência insulínica, obesidade, gordura abdominal, doença renal crônica, alta ingestão de sal, inflamação crônica de baixo grau, estresse oxidativo, dieta inadequada, consumo de álcool, privação social, estresse emocional, sedentarismo e numerosos fatores genéticos como determinantes dos padrões de envelhecimento arterial precoce. ${ }^{7,26,27,30}$

Assim, a idade vascular pode ser maior que a idade cronológica, devido aos fatores de risco cardiovasculares. ${ }^{7,24,26,27,30}$

Em 2010, em revisão sistemática que incluiu 11.902 participantes, foram publicados os valores de referência para avaliação de rigidez arterial em população europeia, de acordo com a idade e a classificação de pressão arterial. Porém, devido a limitações do estudo, os valores de referência para rigidez arterial ainda não forneceram todas as respostas, mas poderiam ser utilizados como uma base sólida para futuras pesquisas e foram um passo importante na direção da padronização da VOPcf. ${ }^{29}$

A relação entre a $\mathrm{VOP} c f$ e a idade não é linear, mas sim quadrática (ou seja, ocorre aumento acelerado), enquanto sua relação com a pressão arterial (PA) é linear. Assim, é difícil propor uma definição única de EVA. Tentativas que não levaram a idade ou PA em consideração foram simplistas. Ter um valor de VOPcf $<10 \mathrm{~m} / \mathrm{s}$ pode corresponder a EVA em indivíduos jovens, enquanto em pessoas mais velhas pode corresponder a SUPERNOVA. 7,26-28,30

A expressão "envelhecimento vascular médio" pode ser usada para qualificar o campo mediano entre os 2 extremos da distribuição da VOPcf, mas ainda não foram definidos limites superior e inferior desse campo mediano, acima dos quais alguns indivíduos têm EVA e abaixo do qual outros têm HVA, respectivamente. Atualmente, é preferível considerar as zonas cinzentas para os limites entre EVA e HVA ao longo da distribuição da VOPcf em populações para uma determinada idade.? (Figuras 1 e 2)

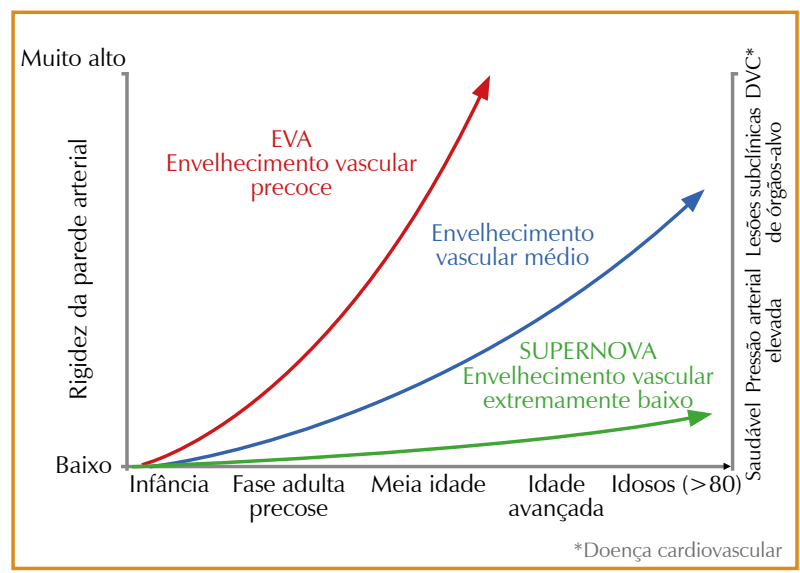

Figura 1. Conceitos envelhecimento vascular. 


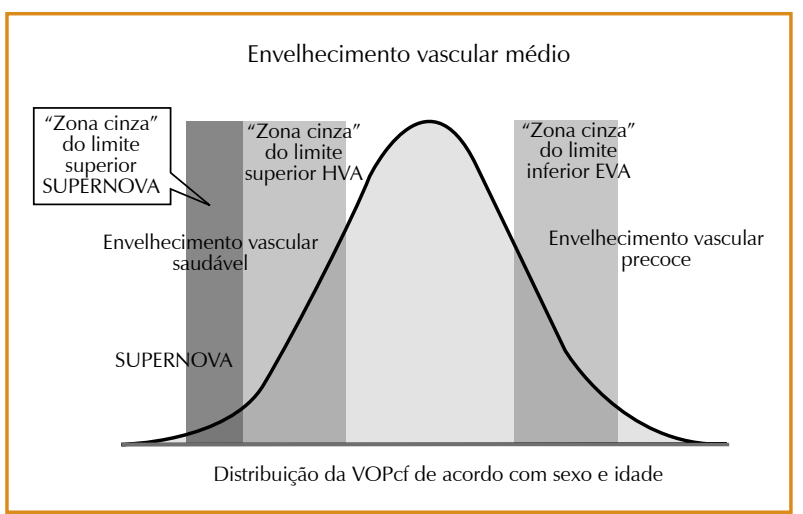

Figura 2. Distribuição da VOPcf. ${ }^{7}$

\section{MÉTODOS PARA AVALIAÇÃO DA RIGIDEZ ARTERIAL}

Antigamente, a avaliação da hemodinâmica central exigia métodos invasivos e procedimentos com um grau alto de complicação, utilizando cateteres intra-arteriais de pressão e dificultando bastante a realização na rotina. ${ }^{16,31}$

Os dispositivos para avaliar a pressão central de forma não invasiva tornaram-se disponíveis no final do século XIX. Os avanços científicos e tecnológicos foram cruciais para permitir que tais dispositivos se tornassem possíveis na prática clínica. ${ }^{16,31,32}$

A rigidez arterial pode ser determinada por três metodologias principais: (1) medindo o atraso de tempo entre dois locais arteriais e estimando a velocidade da onda de pulso a partir da distância entre os locais dividida pelo atraso de tempo; (2) medindo a distensão da artéria e relacioná-la com a pressão pulsátil local; e (3) estimando a rigidez arterial a partir das medidas de pressão do manguito através de modelos de circulação. 7,16,31,32

Atualmente, cinco métodos de avaliação da pressão central são os mais utilizados: dispositivos de tonometria Sphygmocor $^{\circledR}$, HEM-9000AI $^{\circledR}$, Bpro $^{\circledR}$ e APULSE CASP ${ }^{\circledR}$ e os dispositivos oscilométricos com softwares exclusivos Mobil-O-Graph ${ }^{\circledR} \cdot{ }^{16,31,32}$

\section{CENÁRIO ATUAL DA RIGIDEZ ARTERIAL NA PRÁTICA CLÍNICA}

O uso da VOP como biomarcador pode medir o risco geral dos pacientes, identificar danos aos órgãos e facilitar a tomada de decisões clínicas. ${ }^{1}$ No entanto, e apesar da disseminação da medição da VOP por dispositivos não invasivos, sua implementação na prática clínica é sub ótima e restrita a centros terciários. Existe uma lacuna entre o potencial benefício clínico da avaliação da rigidez arterial e a prática de subutilização no mundo real. Isso pode ser atribuído à falta de regulamentação e reembolso das autoridades de saúde e ao custo dos dispositivos utilizados. ${ }^{33}$

\section{REFERÊNCIAS}

1. Vlachopoulos C, Xaplanteris P, Aboyans V, et al. The role of vascular biomarkers for primary and secondary prevention. A position paper from the European Society of Cardiology Working Group on peripheral circulation: Endorsed by the Association for Research into Arterial Structure and Physiology (ARTERY) Society. Atherosclerosis. 2015;241(2):507-32.

2. Conroy RM, Pyörälä K, Fitzgerald AP, et al.; SCORE project group. Estimation of ten-year risk of fatal cardiovascular disease in Europe: the SCORE project. Eur Heart J. 2003;24(11):987-1003.
A fim de reduzir esta lacuna de subutilização da medida da VOP, em 2019, Vlachopoulos e colaboradores validaram o escore de SAGE, em uma população de hipertensos. ${ }^{33} \mathrm{O}$ escore de SAGE é um escore clínico simples que prediz altos valores de VOP com base em variáveis clínicas amplamente disponíveis pode identificar pacientes que devem ter a VOP preferencialmente medida e, portanto, encaminhada para centros terciários ou equipados especificamente, facilitando a disseminação mais ampla do conceito de rigidez aórtica entre os médicos. ${ }^{33}$

Embora haja casos em que alguém possa assumir corretamente que a VOP é alta ou baixa com base no acúmulo de fatores de risco, isso nem sempre pode ser o caso. Além disso, a medição da VOP poderia reclassificar pacientes em outra categoria de risco. O escore SAGE pode ser usado para prever com precisão os níveis elevados de VOP e priorizar sua medição em pacientes hipertensos específicos. ${ }^{33}$

No contexto nacional, está sendo concluído estudo que avaliará a aplicabilidade do escore de SAGE na população brasileira. Seu uso resultará em maior reconhecimento do papel da rigidez aórtica e auxiliará os médicos na sua implementação na prática clínica.

\section{CONCLUSÃO}

A população está envelhecendo rapidamente em todo o mundo, o que levará a uma sobrecarga social e econômica de doenças crônicas associadas à idade, incluindo doenças cardiovasculares. ${ }^{1,2} \mathrm{O}$ rastreio e a gestão de grupos de risco moderado e elevado para eventos cardiovasculares são importantes para reduzir a mortalidade, a morbilidade, e o fardo socioeconômicos associada com doenças cardiovasculares (DCV). ${ }^{8,9,11}$

Eventos cardiovasculares futuros têm sido tradicionalmente previsto usando escores que combina fatores de risco para aterosclerose convencionais, ${ }^{1-5}$ De fato, os valores da velocidade da onda de pulso carotídeo-femoral (VOPcf), como marcador padrão-ouro da rigidez arterial, podem modificar a previsão de risco cardiovascular, especialmente em indivíduos mais jovens com risco intermediário. ${ }^{1-12}$

Assim, a análise do dano vascular baseada apenas nos fatores de risco cardiovasculares clássicos pode falhar na identificação de pessoas excessivamente sensíveis (EVA) ou protegidas contra fatores de risco (HVA), enquanto a VOP representa o dano cumulativo de todos os fatores de risco cardiovascular na parede arterial e portanto, maior especificidade. ${ }^{7}$

\section{CONFLITO DE INTERESSES}

Nenhum potencial conflito de interesse relevante para este artigo foi relatado.

3. Goff DC Jr, Lloyd-Jones DM, Bennett G, et al. 2013 ACC/AHA guideline on the assessment of cardiovascular risk: a report of the American College of Cardiology/American Heart Association Task Force on Practice Guidelines. J Am Coll Cardiol. 2014;63(25 Pt B):2935-2959.

4. Faludi AA, Izar MCO, Saraiva JFK, et al. Atualização da Diretriz Brasileira de Dislipidemias e Prevenção da Aterosclerose - 2017. Arq Bras Cardiol. 2017;109(2 Supl 1):1-76.

5. Biomarkers definitions working group et al. Biomarkers and surrogate 
endpoints: preferred definitions and conceptual framework. Clin Pharmacol Ther. 2001;69(3):89-95.

6. Laurent S, Boutouyrie P, Cunha PG, Lacolley P, Nilsson PM. Concept of Extremes in Vascular Aging. Hypertension. 2019 Aug;74(2):218-228.

7. Laurent S, Boutouyrie P, Asmar R, Gautier I, Laloux B, Guize L, Ducimetiere $\mathrm{P}$, Benetos A. Aortic stiffness is an independent predictor of all-cause and cardiovascular mortality in hypertensive patients. Hypertension. 2001;37(5):1236-41.

8. Boutouyrie P, Tropeano Al, Asmar R, et al. Aortic stiffness is an independent predictor of primary coronary events in hypertensive patients: a longitudinal study. Hypertension. 2002;39(1):10-5.

9. Mitchell GF, Hwang SJ, Vasan RS, et al. Arterial stiffness and cardiovascular events: the Framingham Heart Study. Circulation. 2010;121(4):505-11.

10. Vlachopoulos C, Aznaouridis K, Stefanadis C. Prediction of cardiovascular events and all-cause mortality with arterial stiffness: a systematic review and meta-analysis. J Am Coll Cardiol. 2010;55(13):1318-27.

11. Ben-Shlomo Y, Spears M, Boustred C, et al. Aortic pulse wave velocity improves cardiovascular event prediction: an individual participant metaanalysis of prospective observational data from 17,635 subjects. J Am Coll Cardiol. 2014 Feb 25;63(7):636-646.

12. Laurent $S$, Boutouyrie P. The structural factor of hypertension: large and small artery alterations. Circ Res. 2015;116(6):1007-21.

13. Kaess BM, Rong J, Larson MG, Hamburg NM, Vita JA, Levy D, Benjamin EJ, Vasan RS, Mitchell GF. Aortic stiffness, blood pressure progression, and incident hypertension. JAMA. 2012;308(9):875-81.

14. Koivistoinen T, Lyytikäinen LP, Aatola H, et al. Pulse Wave Velocity Predicts the Progression of Blood Pressure and Development of Hypertension in Young Adults. Hypertension. 2018;71(3):451-6.

15. Brandao AA, Amodeo C, Alcantara C, et al. I. Luso-Brazilian Positioning on Central Arterial Pressure. Arq Bras Cardiol. 2017;108(2): 100-8.

16. Najjar SS, Scuteri A, Shetty $\mathrm{V}$, et al. Pulse wave velocity is an independent predictor of the longitudinal increase in systolic blood pressure and of incident hypertension in the Baltimore Longitudinal Study of Aging. J Am Coll Cardiol. 2008;51(14):1377-83

17. BottoFernando F, Obregon S, Rubinstein F, et al. Frequency of early vascular aging and associated risk factors among an adult population in latin america: the OPTIMO Study. J Hum Hypertens. 2018;32(3):219-27.

18. Sehestedt T, Jeppesen J, Hansen TW, et al. Risk prediction is improved by adding markers of subclinical organ damage to SCORE. Eur Heart J. 2010;31(7):883-91.
19. Mattace-Raso FU, van der Cammen TJ, Hofman A, et al. Arterial stiffness and risk of coronary heart disease and stroke: the Rotterdam Study. Circulation. 2006;113(5):657-63.

20. Mikael LR, Paiva AMG, Gomes MM, et al. Vascular Aging and Arterial Stiffness. Arq Bras Cardiol. 2017 Sep; 109(3):253-258.

21. Brunner-La Rocca HP. Towards applicability of measures of arterial stiffness in clinical routine. Eur Heart J. 2010;31(19):2320-2.

22. Nowak KL, Rossman MJ, Chonchol M, Seals DR. Strategies for Achieving Healthy Vascular Aging. Hypertension. 2018;71(3):389-402.

23. Thijssen $\mathrm{DH}$, Carter $\mathrm{SE}$, Green DJ. Arterial structure and function in vascular ageing: are you as old as your arteries? J Physiol. 2016 Apr 15;594(8):2275-84.

24. Chirinos JA, Segers P, Hughes T, Townsend R. Large-Artery Stiffness in Health and Disease: JACC State-of-the-Art Review. J Am Coll Cardiol. 2019;74(9):1237-63.

25. Safar ME, Asmar R, Benetos A, et al.; French Study Group on Arterial Stiffness. Interaction Between Hypertension and Arterial Stiffness. Hypertension. 2018;72(4):796-805.

26. Cunha PG, Boutouyrie P, Nilsson PM, Laurent S. Early Vascular Ageing (EVA): Definitions and Clinical Applicability. Curr Hypertens Rev. 2017;13(1):8-15.

27. Harper S. Economic and social implications of aging societies. Science. 2014;346(6209):587-591.

28. Kucharska-Newton AM, Stoner L, Meyer ML. Determinants of Vascular Age: An Epidemiological Perspective. Clin Chem. 2019;65(1):108-18.

29. Reference Values for Arterial Stiffness' Collaboration. Determinants of pulse wave velocity in healthy people and in the presence of cardiovascular risk factors: 'establishing normal and reference values'. Eur Heart J. 2010 Oct;31(19):2338-50.

30. Nilsson PM, Lurbe E, Laurent S. The early life origins of vascular ageing and cardiovascular risk: the EVA syndrome. J Hypertens. 2008;26(6):1049-57.

31. McEniery CM, Cockcroft JR, Roman MJ, Franklin SS, Wilkinson IB. Central blood pressure: current evidence and clinical importance. Eur Heart J. 2014;35(26):1719-25.

32. Laurent S, Cockcroft J, Van Bortel L, et al.; European Network for Noninvasive Investigation of Large Arteries. Expert consensus document on arterial stiffness: methodological issues and clinical applications. Eur Heart J. 2006 Nov; $27(21): 2588-605$

33. Xaplanteris P, Vlachopoulos C, Protogerou AD, et al. A clinical score for prediction of elevated aortic stiffness: derivation and validation in 3943 hypertensive patients. Journal of Hypertension. 2019;37(2):339-46. 